\title{
Suppression of Lipid Accumulation in 3T3-L1 Adipocytes by $\alpha$-Tocopheryl Succinate
}

\author{
Dai Majima, ${ }^{a, \#}$ Ryosuke Mitsuhashi, ${ }^{a, \#}$ Misaki Yamasaki, ${ }^{a}$ Kazuaki Kajimoto, ${ }^{b}$ \\ Tatsuya Fukuta, ${ }^{a}$ and Kentaro Kogure ${ }^{*, a}$ \\ ${ }^{a}$ Graduate School of Biomedical Science, Tokushima University; 1-78-1 Shomachi, Tokushima 770-8505, Japan: and \\ ${ }^{b}$ Health Research Institute, The National Institute of Advanced Industrial Science and Technology; 2217-14 Hayashi-cho, \\ Takamatsu, Kagawa 761-0395, Japan.
}

Received July 15, 2020; accepted October 17, 2020

\begin{abstract}
Obesity is a pathological state related to various lifestyle-related diseases, such as diabetes and dyslipidemia, that may be prevented through the development of anti-obesity treatments. Lipid accumulation in cells could be affected by vitamin E ester $\alpha$-tocopheryl succinate (TS), which has various biological activities, such as anti-cancer effect, via activation of cell signaling pathways, although the antioxidative activity of TS is lost due to esterification of the phenolic OH group. In this study, we found for the first time that TS significantly suppressed lipid accumulation in mouse 3T3-L1 adipocytes. TS treatment reduced the amount of triglycerides in the culture medium, and inhibited activity of glycerol-3-phosphate dehydrogenase, a marker of lipid synthesis. Furthermore, TS accelerated lipolysis. Treatment of adipocytes with TS for $24 \mathrm{~h}$ induced no significant cytotoxicity. In TS-treated cells, phosphorylation of Akt, which is involved in fatty acid synthesis via sterol regulatory element-binding proteins (SREBP), was prevented, while levels of phosphorylated protein kinase A (PKA) did not change. Taken together, these results suggest that vitamin E ester TS can suppress lipid accumulation in adipocytes by regulating lipid metabolic cell signaling.
\end{abstract}

Key words tocopheryl succinate; adipocyte; lipolysis; anti-obesity

\section{INTRODUCTION}

Obesity is a serious public health problem worldwide and is a main risk factor for various diseases including arterial hypertension, dyslipidemia, and type 2 diabetes mellitus. ${ }^{1)}$ Exercise and dietary restrictions are effective for reducing the risk of obesity, but many individuals find such approaches challenging over the long-term. As such, medications to lower the incidence of obesity are needed. Several compounds such as phentermine/topiramate, lorcaserin, naltrexone/bupropion and liraglutide, have been approved by the United States Food and Drug Administration to treat obesity. ${ }^{2)}$ The norepinephrine transporter inhibitor phentermine suppresses appetite by activating hypothalamic proopiomelanocortin arcuate nucleus neurons. Topiramate is an antiepileptic drug that also suppresses appetite by modulating activity of voltage-gated ion channels. Lorcaserin is a centrally acting anorexic agent, whereas the opioid antagonist naltrexone suppresses appetite. Bupropion, which is an atypical antidepressant, induces an anorexic effect and the glucagon like peptide-1 receptor agonist liraglutide induces weight loss by suppressing appetite and delaying gastric emptying. The mechanisms of these medicines mainly involve appetite suppression. However, once formed, reducing amounts of adipose tissue is difficult, and these problems can produce side effects by acting on feeding centers in the brain. As such, medicines that can reduce the amount of existing adipose tissue are needed.

We propose $\alpha$-tocopheryl succinate (TS; Fig. 1a), the succinate ester form of the well-known antioxidant $\alpha$-tocopherol $(\alpha-\mathrm{T})$, as a novel candidate anti-obesity medicine that could

\footnotetext{
\# These authors contributed equally to this work.

reduce the volume of adipose tissue. TS lacks antioxidative activity but retains various biological activities, such as inhibition of cholinesterase, inhibition of nuclear factor-kappa $\mathrm{B}$ $(\mathrm{NF}-\kappa \mathrm{B})$ activation, and enhancement of lipopolysaccharideinduced nitric oxide production, in addition to anticancer effects. $^{3)}$ In particular, TS is thought to activate the cell signaling enzyme protein kinase $\mathrm{C}(\mathrm{PKC})^{4)}$ through direct interactions. ${ }^{5)}$ Since adipogenesis is regulated by various cell signaling pathways, ${ }^{6,7)}$ we expected that TS could affect lipid accumulation by regulating cell signaling pathways in adipocytes.

In the present study, we examined the effect of TS on lipid accumulation in mouse 3T3-L1 adipocytes. However, the water solubility of TS is low due to its hydrophobicity and thus TS tends to aggregate during long incubations in cell culture. In this study, we prepared liposomes containing TS that avoids TS aggregation in culture medium. We treated adipocytes with TS and examined lipid accumulation by these cells in different cell states including differentiation and maturation. We also quantified the amount of triglyceride in the culture medium. To determine the mechanism by which TS affects lipid accumulation, levels of glycerol-3-phosphate dehydrogenase activity as a marker of lipid synthesis, and glycerol secretion via lipolysis were quantified. Cytotoxicity of TS-L treatment for $24 \mathrm{~h}$ was measured and phosphorylation levels of proteins involved in lipid metabolism signaling pathways, such as protein kinase A (PKA) and the serine/ threonine-protein kinase Akt, were evaluated by Western blotting. The results were examined to obtain the information regarding the mechanism of TS-mediated effects on lipid accumulation in adipocytes. 
a

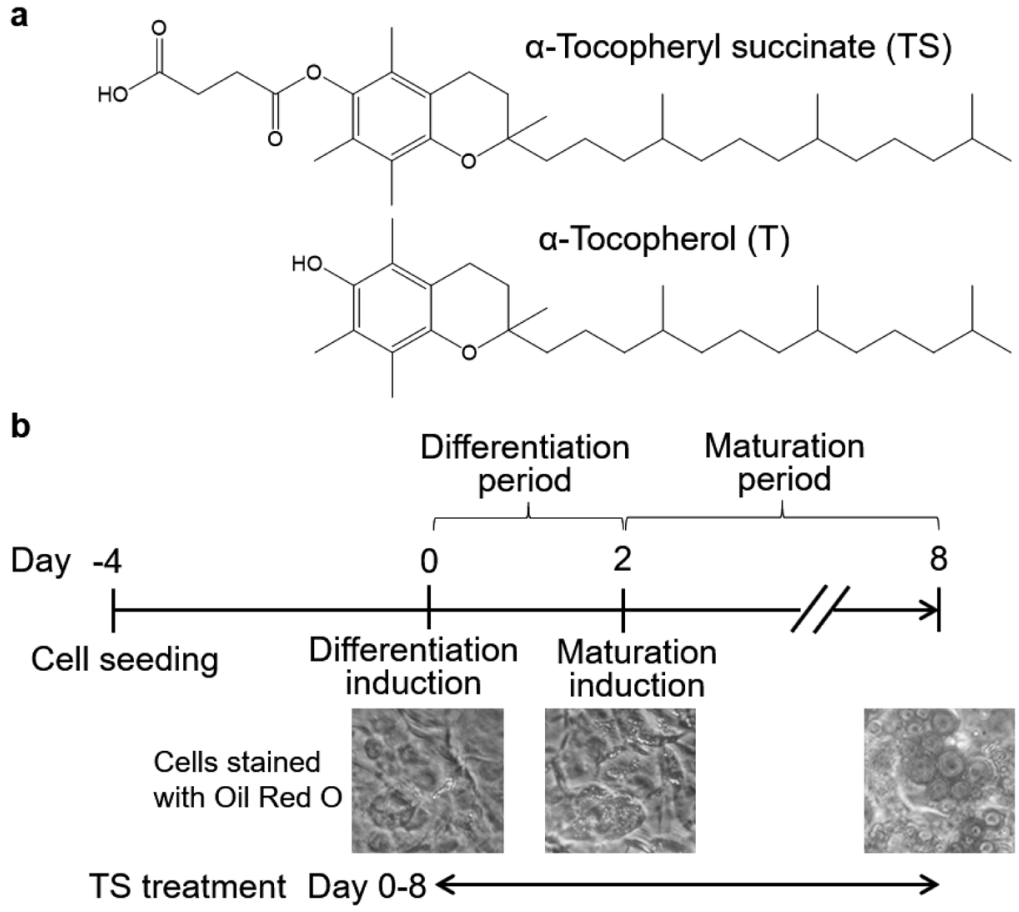

Fig. 1. (a) Chemical Structures of $\alpha$-Tocopheryl Succinate (TS) and $\alpha$-Tocopherol (T), and (b) Timeline for Differentiation/Maturation Induction and 3T3-L1 Adipocyte Treatments

\section{MATERIALS AND METHODS}

Materials TS was purchased from Focus Biomolecules (Plymouth Meeting, PA, U.S.A.). Egg phosphatidylcholine (EPC) and Oil Red O were from Sigma-Aldrich (St. Louis, MO, U.S.A.). $\alpha$-Tocopherol was kindly provided by Eisai (Tokyo, Japan). Other reagents were of the highest grade commercially available. Mouse fibroblast Swiss 3T3 cells were obtained from American Type Culture Collection. Mouse pre-adipocyte 3T3-L1 cells were purchased from JCBR (Tokyo, Japan).

Cell Culture of 3T3-L1 Adipocytes 3T3-L1 cells $\left(2 \times 10^{5}\right.$ cells) were seeded on a $35 \mathrm{~mm}$ dish and cultured for $96 \mathrm{~h}$ at $37^{\circ} \mathrm{C}$ in a $\mathrm{CO}_{2}$ incubator to confluency. Then, the culture medium was changed to a differentiation induction medium containing $467.5 \mu \mathrm{M}$ isobutyl methyl xanthine, $0.93 \mu \mathrm{M}$ dexamethasone and $1.637 \mu \mathrm{M}$ insulin (Day 0 ). After $48 \mathrm{~h}$ culture (Day 2) to induce differentiation, the medium was changed to a maturation medium containing $1.637 \mu \mathrm{M}$ insulin and $3.72 \mathrm{mg} / \mathrm{L}$ D-biotin. The maturation medium was changed every $2 \mathrm{~d}$ until Day 8.

Liposome Preparation A chloroform solution containing $0.1 \mathrm{~mL}$ of $0.1 \mathrm{M}$ TS ethanol solution (or tocopherol solution) and $49 \mu \mathrm{L} 0.1 \mathrm{~g} / \mathrm{mL}$ EPC in chloroform in a glass test tube was evaporated to dryness under a nitrogen gas stream. The dried lipid film was hydrated with $0.2 \mathrm{~mL}$ phosphate buffered saline (PBS) solution containing $8 \mu \mathrm{L} 1 \mathrm{M} \mathrm{NaOH}$. After $10 \mathrm{~min}$ incubation at room temperature, the glass test tube was sonicated in an ultrasonic bath for $20 \mathrm{~min}$ to form liposomes. The size and surface charge of liposomes were measured using Zetasizer Nano (Malvern Panalytical Ltd., U.K.). Liposome diameters and zeta-potentials are summarized in Table 1.

Evaluation of Lipid Accumulation Lipid accumulation by adipocytes was evaluated by Oil Red O staining. ${ }^{8)}$ The cells were fixed with $10 \%$ neutral buffered formalin solution,
Table 1. Characteristics of Liposomes

\begin{tabular}{lcc}
\hline \hline Liposome composition & Diameter $(\mathrm{nm})$ & Zeta-potential $(\mathrm{mV})$ \\
\hline EPC only & $217.1 \pm 54.2$ & $-2.21 \pm 2.13$ \\
EPC + tocopherol & $200.2 \pm 28.7$ & $-3.01 \pm 0.18$ \\
EPC + TS & $176.1 \pm 28.5$ & $-40.9 \pm 2.90$ \\
\hline
\end{tabular}

The values are means \pm standard deviation (S.D.) $(n \geq 3)$.

washed once with $60 \%$ isopropanol and incubated with $1 \mathrm{~mL}$ $60 \%$ Oil Red O solution for $20 \mathrm{~min}$ at room temperature for lipid staining. The fixed and stained cells were then washed once with $60 \%$ isopropanol and twice with distilled water before $1 \mathrm{~mL}$ of distilled water was added to the dish for observation of Oil Red O staining with a BIOREVO microscope (KEYENCE Co., Osaka, Japan). To extract Oil Red O, the cells in the culture dish were dried and $3 \mathrm{~mL} 100 \%$ isopropanol was added. The absorbance of the extracted solution at $540 \mathrm{~nm}$ was then measured.

Measurement of Time-Dependent Changes in Triglyceride in Cell Culture Medium To examine lipid synthesis following TS treatment, the amount of triglycerides in culture medium collected every $2 \mathrm{~d}$ was measured using the commercially available assay kit LabAssay ${ }^{\mathrm{TM}}$ Triglyceride (FUJIFILM Wako Pure Chemical Corporation, Osaka, Japan).

Evaluation of Lipid Synthesis and Lipolysis Activities To evaluate lipid synthesis activity, the activity of glycerol3-phosphate dehydrogenase (GPDH) in cells was measured. Adipocytes treated with liposomes during Day 0 to 8 were washed with PBS, and the cytoplasmic fraction was extracted according to the manufacturer's recommendations for the GPDH Assay Kit (TaKaRa Bio Inc., Shiga, Japan) used to measure GPDH activity. To evaluate lipolysis activity, 3T3-L1 cells were cultured until Day 4 to allow lipid accumulation before the indicated liposome formulation was added to the 

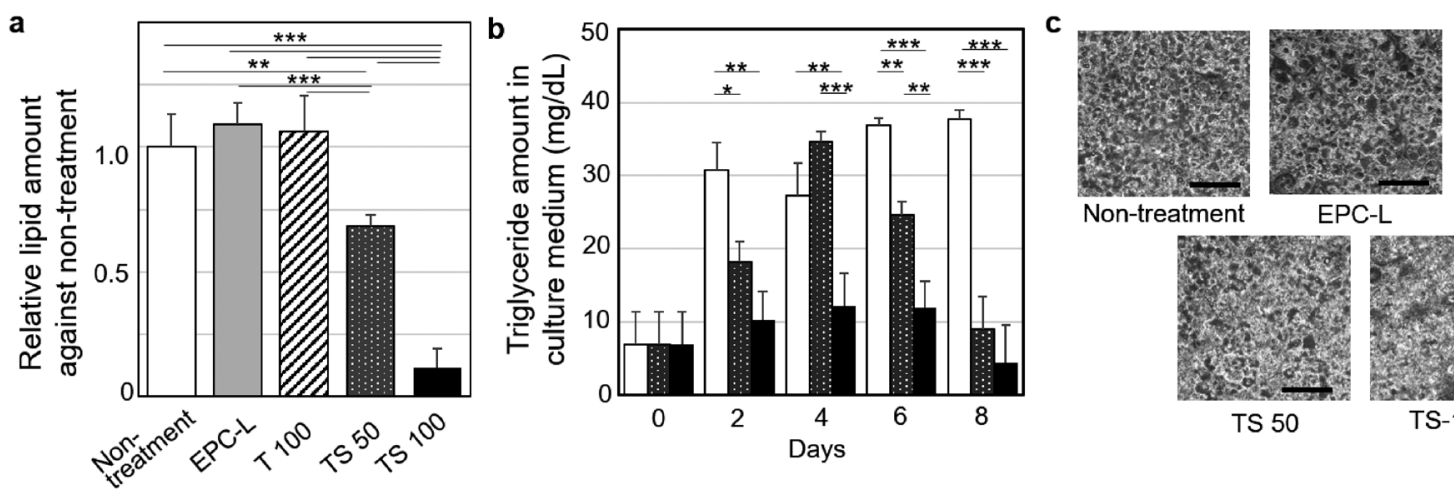
EPC-L
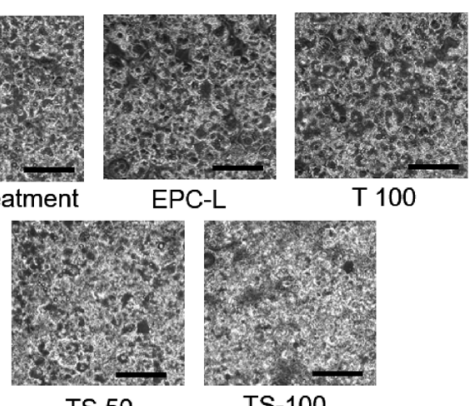

T 100

Fig. 2. Effect of TS on Lipid Accumulation by 3T3-L1 Cells, and Amount of Triglycerides in Culture Medium

(a) Adipocytes were treated with control liposomes (EPC-L), liposomes containing T or liposomes containing $\alpha$-TS. The final concentration of T was 50 (TS 50) and $100 \mu \mathrm{M}$ (TS 100). The amount of accumulated lipid was quantified by measuring absorbance of Oil Red O extracted from adipocytes on Day 8 . Values are the average of the results for at least three experiments; **p<0.01, $* * * p<0.001$. (b) The amount of triglycerides in the culture medium was measured every $2 \mathrm{~d}$ following treatment of cells with 50 (polka dot bar) and $100 \mu \mathrm{M}$ (black bar) of TS. Values are the average of the results for at least three experiments; $* p<0.05, * * p<0.01, * * * p<0.001$. (c) Microscopic images of the 3T3-L1 cells stained with Oil Red O on Day 8.
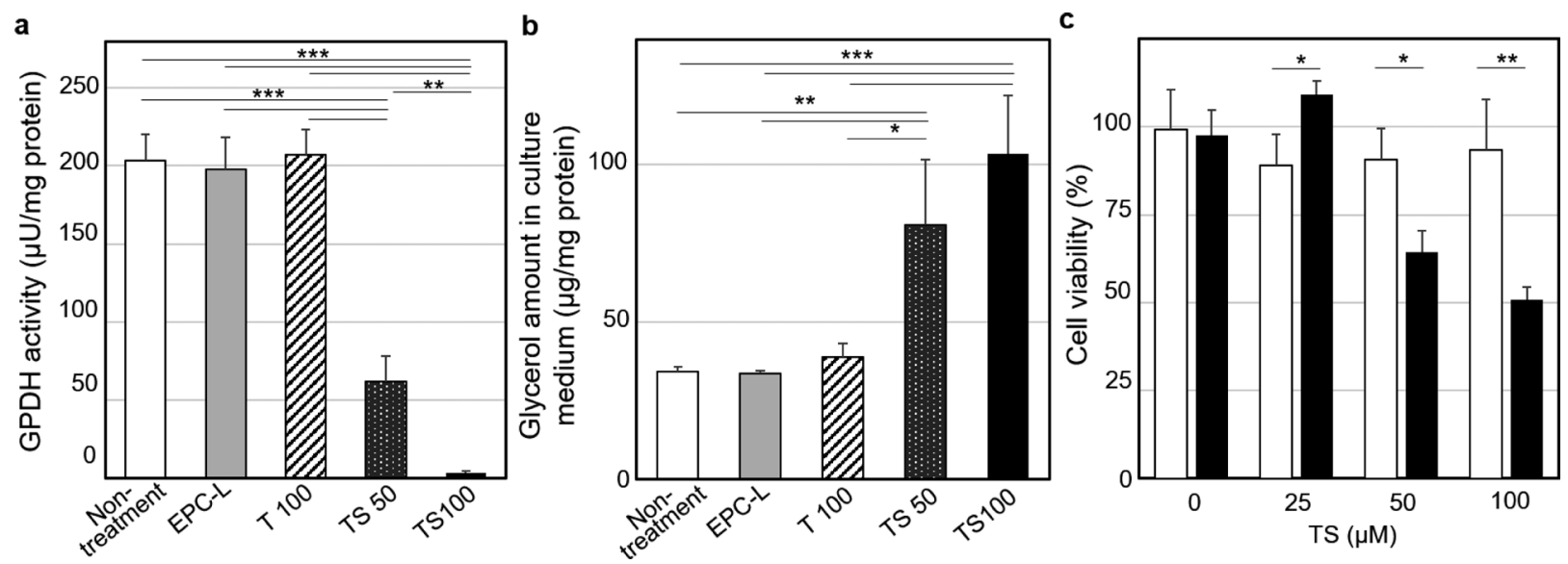

Fig. 3. Effect of TS on GPDH Activity, Amount of Glycerol in Culture Medium and Viabilities of Adipocyte and Fibroblast

(a) Adipocytes were treated with EPC-L, T, or TS between Day 0 to 8 , and then GPDH activity was measured. The final concentrations of T and TS were 100 (T 100), 50 (TS 50) and $100 \mu \mathrm{M}$ (TS 100). Values are the average of the results for at least three experiments; $* * p<0.01, * * * p<0.001$. (b) Adipocytes were treated with EPC-L, $\mathrm{T}$ or TS on Day 4 for two days before the amount of glycerol in the culture medium was measured. The final concentrations of T and TS were 100 (T 100), 50 (TS 50 ) and $100 \mu \mathrm{M}$ (TS 100). Values are the average of the results for at least three experiments; ${ }^{*} p<0.05,{ }^{* *} p<0.01, * * * p<0.001$. (c) Mouse 3 T3-L1 adipocytes (white bar) and mouse Swiss 3 T3 fibroblasts (black bar) were treated with various concentrations of TS for $24 \mathrm{~h}$. Values are the average of the results for at least three experiments; $* p<0.05, * * p<0.01$.

cells. After $48 \mathrm{~h}$, the culture medium was collected, and the amount of glycerol was measured using a Glycerol Colorimetric Assay Kit (Cayman Chemical, MI, U.S.A.).

Measurement of Cell Viability Swiss $3 \mathrm{~T} 3$ cells or 3T3-L1 cells $\left(1.5 \times 10^{5}\right.$ cells/well $)$ were seeded in 6 -well plates and cultured for $24 \mathrm{~h}$ at $37^{\circ} \mathrm{C}$ in a $\mathrm{CO}_{2}$ incubator. The cells were treated with the indicated liposome formulation for $24 \mathrm{~h}$ at $37^{\circ} \mathrm{C}$. Then, the cells were treated with trypsin and collected for measurement of cell viability using the trypan blue staining method. Cell viability is the percentage obtained by dividing the number of cells stained with trypan blue by the total number of cells.

Evaluation of Protein Kinase A and Akt Phosphorylation Phosphorylation of PKA and Akt was evaluated by Western blotting. Cells were collected on Day 8 with a cell scraper and solubilized in lysis buffer. The protein content of the cell lysate was measured, and equal amounts of cellular proteins were subjected to Western blotting with anti-mouse Phospho-PKA C (Thr197) (D45D3). Rabbit monoclonal antibody (Cell Signaling Technology, Danvers, MA, U.S.A.) or anti-mouse Phospho-Akt (Ser473, D9E) rabbit monoclonal antibody (Cell Signaling Technology) was used as the primary antibody. Band intensity was quantified using ImageJ.

Statistical Analysis Statistical significance was determined using one-way ANOVA, followed by Tukey's honestly significant difference test. $p$ Values $<0.05$ were considered significant.

\section{RESULTS}

Effect of TS on 3T3-L1 Cell Lipid Accumulation To study the effect of TS on lipid accumulation, 3T3-L1 adipocytes were treated with liposomes containing TS across Days 0-8 (Fig. 1b) and the amount of lipids in the cells was quantified by Oil Red $\mathrm{O}$ staining. Egg phosphatidylcholine (EPC) liposomes without TS (EPC-L) and liposomes containing tocopherol (T), obtained by hydrolysis of TS, did not affect lipid accumulation by the cells (Fig. 2a). The amount of accumulated lipid was significantly reduced by $50 \mu \mathrm{M}$ TS (TS 50). Furthermore, treatment with TS 100 induced a dramatic decrease in the amount of intracellular lipids (Fig. 2a).

Effect of TS on Triglyceride Secretion to Cell Culture 
a

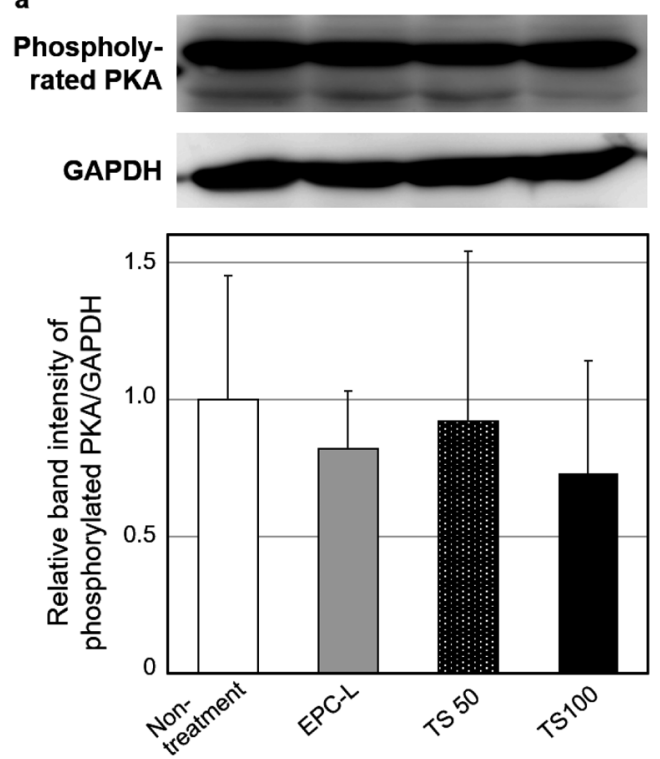

b
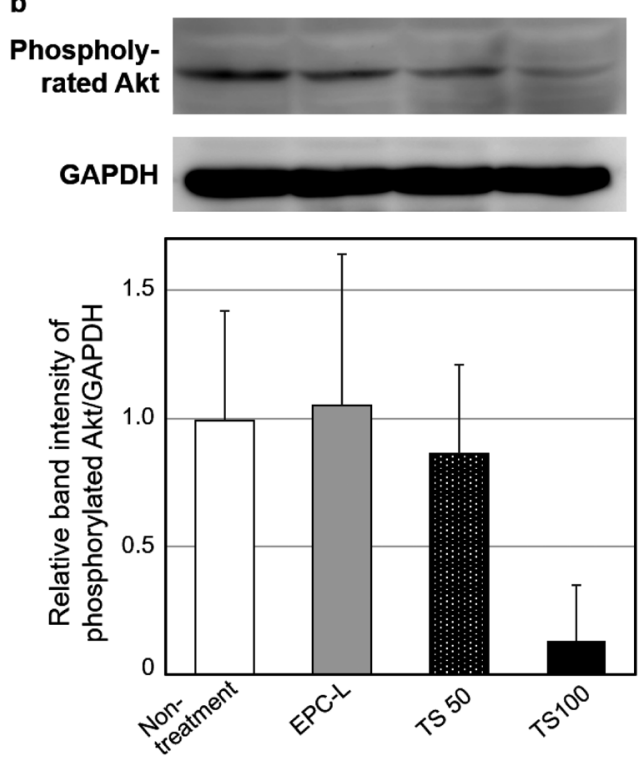

Fig. 4. Effect of TS on Phosphorylation of PKA and Akt

Phosphorylation of (a) PKA and (b) Akt was detected by Western blotting of lysates from adipocytes treated with TS across Day 0 to 8 . Band intensity was quantified using ImageJ, and relative band intensity was obtained by calculation using glyceraldehyde 3-phosphatase dehydrogenase (GAPDH). Values are the average of the results for at least three experiments.

Medium The amount of triglyceride in culture medium of untreated 3T3-L1 adipocytes exhibited time-dependent increases (Fig. 2b). Treatment with TS 50 suppressed the rate of triglyceride increase during Days $0-4$, and thereafter the amounts were significantly reduced relative to untreated cells. Treatment with TS 100 almost completely suppressed increases in the amount of triglyceride in the culture medium (Fig. 2b). This result aligns with the results for Oil Red O staining (Fig. 2c).

Effect of TS Treatment on Lipogenesis and Lipolysis by 3T3-L1 Adipocytes To understand the mechanism by which TS prevents lipid accumulation in adipocytes, we examined the effects of TS treatment on the activity of GPDH as a measure of lipogenesis and the amount of glycerol in culture medium as a marker of lipolysis. Treatment of adipocytes with TS, particularly TS 100 , during Days 0-8 significantly reduced GPDH activity (Fig. 3a). The amount of glycerol in culture medium increased with TS treatment during Days 4-6, after accumulation of lipids by adipocytes (Fig. 3b). The amount of glycerol for TS 100-treated cells was 3-fold higher than that of non-treated cells.

Effect of TS Treatment on Cell Viability Since TS could have an anti-cancer effect due to its ability to induce apoptosis, we next examined the effect of TS on viability of both mouse fibroblast Swiss 3T3 cells and 3T3-L1 adipocytes after treatment with various amounts of TS for $24 \mathrm{~h}$. We observed dose-dependent decreases in Swiss $3 \mathrm{~T} 3$ cell viability, but the viability of 3T3-L1 adipocytes was not affected by TS, even at $100 \mu \mathrm{M}$ (Fig. 3c).

Effect of TS on Cell Signaling Pathways Related to Lipid Metabolism To further clarify the mechanism of TS action, we assessed the activity of PKA and Akt, which are involved in lipid metabolism cell signaling pathways, ${ }^{7}$ by Western blotting to detect phosphorylation levels of these proteins. Control EPC-L did not affect the amount of phosphorylated PKA (Fig. $4 a)$. In contrast to our expectation, TS treatment also did not induce significant changes in the amount of phosphorylated PKA (Fig. 4a). The amount of phosphorylated Akt tended to decrease with $100 \mu \mathrm{M}$ TS, although this decrease was not statistically significant (Fig. 4b).

\section{DISCUSSION}

We previously reported liposomes containing TS for anticancer therapies. ${ }^{9-11)}$ Liposomes containing TS had higher activity than ethanol solutions of $\mathrm{TS}^{11)}$ suggesting that the liposomes improved delivery efficiency of TS to active sites in the cell cytoplasm. ${ }^{11)}$ In addition, liposomes reduce the likelihood of TS aggregation that can arise during long incubations due to the hydrophobic nature of this compound. In this study, the liposomes containing TS was used to examine the effects of TS on 3T3-L1 mouse adipocyte cells.

Here we showed that TS significantly reduced the amount of lipid accumulated by 3T3-L1 adipocytes (Fig. 2a) relative to control liposomes without TS (EPC-L) and liposomes containing tocopherol, which is produced by hydrolysis of TS. This result indicates that the reduction of lipid amount is due to TS itself. Increases in triglyceride in the culture medium of 3T3-L1 adipocytes were also suppressed by TS treatment (Fig. 2b). Previous studies reported that vitamin E family tocotrienols inhibited lipid accumulation through the prevention of adipocyte differentiation. ${ }^{12,13)}$ Additionally, recent reports showed that vitamin $\mathrm{E} \alpha$-tocopherol and $\delta$-tocopherol induce differentiation of preadipocytes to beige adipocytes, which are thermogenic and consume lipids to generate heat. Beige adipocytes exhibit up-regulated gene expression of peroxisome proliferator-activated receptor $\gamma$ coactivator- $1 \alpha(\mathrm{PGC1}-\alpha)$ and uncoupling protein 1 (UCP1) that occurs upon activation of p38 mitogen-activated protein kinase (MAPK). ${ }^{14,15)}$

On the other hand, TS treatment of 3T3-L1 adipocytes significantly reduced GPDH activity and increased the amount of glycerol in the culture medium, respectively (Figs. 3a, b), 
suggesting that TS can prevent lipid accumulation not only by inhibiting lipid synthesis, but also by accelerating lipolysis. Therefore, TS could be a candidate anti-obesity therapeutic that could enhance lipolysis and in turn reduce adipose tissue that has formed.

Even at the highest concentrations, TS did not affect adipocyte cell viability, whereas that of fibroblasts decreased dosedependently (Fig. 3c). TS was previously shown to damage fibroblasts and various cancer cells, ${ }^{3,11)}$ but here we showed that TS is not toxic to adipocytes, at least under the conditions examined. This result indicates that the inhibitory effect of TS on lipid accumulation was not due to cytotoxic effects. In Swiss 3T3 cells, cell viability slightly increased at TS $25 \mu \mathrm{M}$. Presumably, activation of cell signaling, such as PKC, by TS stimulated cell proliferation at this concentration.

PKA is known to accelerate lipolysis through phosphorylation of hormone-sensitive lipase (HSL). ${ }^{7)}$ Here we showed that levels of PKA phosphorylation were not affected by TS treatment (Fig. 4a), although the amount of lipolysis did increase (Fig. 3b). Taken together, these findings suggest that PKA is not a target of TS. On the other hand, Akt phosphorylation was inhibited by TS (Fig. 4b). Akt activates sterol regulatory element-binding protein (SREBP)-1 that in turn regulates fatty acid synthesis. ${ }^{16)}$ Although TS did affect Akt phosphorylation levels, whether it acts directly on Akt is unclear. In addition, the mechanism by which TS inhibits lipid synthesis requires additional investigation.

In conclusion, in this study we demonstrate for the first time that vitamin E ester TS can prevent lipid accumulation in mouse 3T3-L1 adipocytes. TS inhibited lipid synthesis and promoted lipolysis of adipocytes. Taken together, these results suggest that TS could be a novel candidate anti-obesity medicine. Cytotoxicity of TS toward adipocytes was not observed, even though TS treatment decreased the viability of both fibroblasts and cancer cells. As such, future studies should examine strategies to reduce the overall cytotoxic potential of TS.

In terms of liposomal formulation of TS, previous studies reported that liposomes administered via intravenous injection can accumulate in adipose tissue similarly to that seen for tumor tissues via enhanced permeability and retention (EPR) effect. ${ }^{17)}$ Drug delivery technologies, such as PEGylation of TS liposomes, could be used to reduce cytotoxicity of TS towards normal cells by extending the blood circulation time that facilitates passive targeting to adipose tissue. Cytotoxicity might also be reduced by modifying the chemical structure of TS based on this mechanistic study. Together, such modifications could enhance the usefulness of TS as an anti-obesity agent.

Acknowledgments This research was supported by the Research Program for the Development of Intelligent Tokushima Artificial Exosome (iTEX) from Tokushima University.

Conflict of Interest The authors declare no conflict of interest.

\section{REFERENCES}

1) De Lorenzo A, Gratteri S, Gualtieri P, Cammarano A, Bertucci P, Di Renzo L. Why primary obesity is a disease? J. Transl. Med., 17, 169 (2019).

2) Montan PD, Sourlas A, Olivero J, Silverio D, Guzman E, Kosmas CE. Pharmacologic therapy of obesity: mechanisms of action and cardiometabolic effects. Ann. Transl. Med., 7, 393 (2019).

3) Majima D, Mitsuhashi R, Fukuta T, Tanaka T, Kogure K. Biological functions of $\alpha$-tocopheryl succinate. J. Nutr. Sci. Vitaminol., 65 (Supplement), S104-S108 (2019).

4) Kogure $\mathrm{K}$, Morita M, Hama S, Nakashima S, Tokumura A, Fukuzawa K. Enhancement by alpha-tocopheryl hemisuccinate of nitric oxide production induced by lypopolysaccharide and interferon-gamma through the upregulation of protein kinase $\mathrm{C}$ in rat vascular smooth muscle cells. Eur. J. Biochem., 269, 2367-2372 (2002).

5) Kogure K, Hama S, Goto S, Munakata T, Tokumura A, Fukuzawa K. $\alpha$-Tocopheryl succinate activates protein kinase $\mathrm{C}$ in cellular and cell-free systems. J. Nutr. Sci. Vitaminol., 49, 310-314 (2003).

6) Chang E, Kim CY. Natural products and obesity: a focus on the regulation of mitotic clonal expansion during adipogenesis. Molecules, 24, 1157 (2019).

7) Zechner R, Zimmermann R, Eichmann TO, Kohlwein SD, Haemmerle G, Lass A, Madeo F. Fat signals-lipases and lipolysis in lipid metabolism and signaling. Cell Metab., 15, 279-291 (2012).

8) Hasan M, Tarashima N, Fujikawa K, Ohgita T, Hama S, Tanaka T, Saito H, Minakawa N, Kogure K. The novel functional nucleic acid iRed effectively regulates target genes following cytoplasmic delivery by faint electric treatment. Sci. Technol. Adv. Mater., 17, 554-562 (2016).

9) Kogure K, Manabe S, Hama S, Tokumura A, Fukuzawa K. Potentiation of anti-cancer effect by intravenous administration of vesiculated $\alpha$-tocopheryl hemisuccinate on mouse melanoma in vivo. Cancer Lett., 192, 19-24 (2003).

10) Kogure K, Manabe S, Suzuki I, Tokumura A, Fukuzawa K. Cytotoxicity of $\alpha$-tocopheryl succinate, malonate and oxalate in normal and cancer cells in vitro and their anti-cancer effects on mouse melanoma in vivo. J. Nutr. Sci. Vitaminol., 51, 392-397 (2005).

11) Hama S, Utsumi S, Fukuda Y, Nakayama K, Okamura Y, Tsuchiya H, Fukuzawa K, Harashima H, Kogure K. Development of a novel drug delivery system consisting of an antitumor agent tocopheryl succinate. J. Control. Release, 161, 843-851 (2012).

12) Uto-Kondo H, Ohmori R, Kiyose $\mathrm{C}$, Kishimoto $\mathrm{Y}$, Saito H, Igarashi O, Kondo K. Tocotrienol suppresses adipocyte differentiation and Akt phosphorylation in 3T3-L1 preadipocytes. J. Nutr., 139, 51-57 (2009).

13) Burdeos GC, Nakagawa K, Abe T, Kimura F, Miyazawa T. Tocotrienol modulates crucial lipid metabolism-related genes in differentiated 3T3-L1 preadipocytes. Food Funct, 5, 2221-2227 (2014).

14) Tanaka-Yachi R, Takahashi-Muto $\mathrm{C}$, Adachi $\mathrm{K}$, Tanimura $\mathrm{Y}$, Aoki Y, Koike T, Kiyose C. Promoting effect of $\alpha$-tocopherol on beige adipocyte differentiation in 3T3-L1 cells and rat white adipose tissue. J. Oleo Sci., 66, 171-179 (2017).

15) Tanaka-Yachi R, Shirasaki M, Otsu R, Takahashi-Muto C, Inoue $\mathrm{H}$, Aoki Y, Koike T, Kiyose C. $\delta$-Tocopherol promotes thermogenic gene expression via PGC-1 $\alpha$ upregulation in 3T3-L1 cells. Biochem. Biophys. Res. Commun., 506, 53-59 (2018).

16) Krycer JR, Sharpe LJ, Luu W, Brown AJ. The Akt-SREBP nexus: cell signaling meets lipid metabolism. Trends Endocrinol. Metab., 21, 268-276 (2010).

17) Hossen MN, Kajimoto K, Akita H, Hyodo M, Harashima H. Vascular-targeted nanotherapy for obesity: unexpected passive targeting mechanism to obese fat for the enhancement of active drug delivery. J. Control. Release, 163, 101-110 (2012). 